\title{
Stabilization of the moving front solution to the reaction-diffusion-advection problem
}

\author{
Natalia LEVASHOVA ${ }^{1}$, NIKOLAY NEFEDOV ${ }^{2}$, Andrey Orlov ${ }^{1}$, and Elena Polezhaeva ${ }^{1}$ \\ ${ }^{1}$ Moskovskij gosudarstvennyj universitet imeni M V Lomonosova \\ ${ }^{2}$ Moskovskij gosudarstvennyj universitet imeni M V Lomonosova Fiziceskij fakul'tet
}

May 5, 2020

\section{Introduction}

Propagating fronts are characteristic for many physical phenomena. In case of reaction-diffusion-advection processes, these can be combustion or strain fronts. Solutions having large gradients to problems of this type also arise in nonlinear acoustics. Such problems include, for example, the Burgers equation, as well as equations with modular nonlinearity(Rudenko, 2017; Nefedov \& Rudenko, 2018). The stationary reactiondiffusion-advection equations can be used for modelling of wind field distribution in the presence of plant heterogeneity(Levashova et al., 2017; Levashova et al., 2017). The domain where the solution has a large gradient is called the internal transition layer. The numerical implementation of solutions to problems with internal transition layers requires a preliminary analysis of the existence conditions and stability $(\mathrm{Quinn}$, 2015; Kopteva \& Stynes, 2011; O'Riordan \& Quinn, 2011; Lukyanenko et al., 2018; Lukyanenko et al., 2019; Lukyanenko et al., 2019). In particular, for the numerical solution of some applied problems, the calculation method for establishing is often used, when the solution of the boundary value problem for the elliptic equation is found numerically as the solution of the corresponding initial-boundary value problem for the parabolic equation over a sufficiently long period of time. To implement this method, information on the asymptotic stability and the domain of attraction of the stationary solution is needed.

In this paper, we consider the initial-boundary-value problem for reaction-diffusion-advection equation and the question of its moving front type solution stabilizing over an infinitely large time interval to the solution of the corresponding stationary problem. The existence of moving front solution is investigated in(Antipov et al., 2014). The existence conditions of an asymptotically stable solution to the stationary problem are known from(Vasil'eva, 1995). To prove the stabilization theorem, in this paper we use the method of upper and lower solutions, which for this class of problems is justified in(Kazdan \& Kramer, 1978; Wang, 1998). The main idea of the proof is to show that the upper and lower solutions of the initial-boundary-value problem on an asymptotically large time interval fall into the attraction domain of the stationary solution. The upper and lower solutions with large gradients in the region of the internal transition layer are constructed according to the asymptotic method of differential inequalities(Nefedov, 1995; Vasil'eva et al., 2010) as modifications of asymptotic approximations of the solutions to these problems in a small parameter. A small parameter here is the width of the inner transition layer with respect to the width of the front propagation region.

The study conducted in this work gives an answer about non-local domain of attraction of the stationary solution. In addition, an estimate of the time interval is obtained in which the solution of the front type falls into the local domain of attraction of the stationary solution, that is, in fact, the criterion for the numerical solution stationing. 


\section{Problem statement}

We consider the following initial-boundary-value problem for the reaction-diffusion-advection equation:

$$
\begin{gathered}
\varepsilon \frac{\partial^{2} v}{\partial x^{2}}-\frac{\partial v}{\partial t}=A(v, x) \frac{\partial v}{\partial x}+B(v, x), \quad x \in(0 ; 1), \quad t>0 \\
v(0, t)=u^{0}, \quad v(1, t)=u^{1}, t>0, \quad v(x, 0)=v_{\text {init }}(x, \varepsilon), x \in[0 ; 1] .
\end{gathered}
$$

Here $A(v, x), B(v, x) \in C^{3}\left(I_{v} \times[0 ; 1]\right)$ where $I_{v}$ is valid interval of variable $v, \varepsilon>0$ is a small parameter.

If there is a stationary solution $u_{\varepsilon}(x)$ to the problem 1 , it can be defined as a solution to a boundary value problem

$$
\varepsilon \frac{d^{2} u}{d x^{2}}=A(u, x) \frac{d u}{d x}+B(u, x), \quad x \in(0 ; 1), \quad u(0)=u^{0}, \quad u(1)=u^{1}
$$

with the same functions $A$ and $B$ and values $u^{0,1}$, that in statement1.

We assume some propositions.

Let the equation $A(v, x) \frac{d v}{d x}+B(v, x)=0$ with the additional condition $v(0)=u^{0}$ has a solution $v=\varphi^{(-)}(x)$ on the segment $[0 ; 1]$ and with additional condition $v(1)=u^{1}$ has a solution $v=\varphi^{(+)}(x)$, and the inequalities hold:

$$
\varphi^{(-)}(x)<\varphi^{(+)}(x), \quad A\left(\varphi^{(-)}(x), x\right)>0, \quad A\left(\varphi^{(+)}(x), x\right)<0, \quad x \in[0 ; 1] .
$$

The existence conditions of moving front solution to problem1 are formulated in(Antipov et al., 2014) in assumption that the initial function $v_{\text {init }}(x, \varepsilon)$ has already a front form. At each instant of time the front is localized in a vicinity of point $\hat{x}(t) \in(0 ; 1)$, to the left of this vicinity the solution is close to function $\varphi^{(-)}(x)$, and to the right - to function $\varphi^{(+)}(x)$.

In(Vasil'eva, 1995) the existence conditions of Lyapunov asymptotically stable stationary solution with internal transition layer to problem1 are obtained. We consider the transition layer to be localized in a vicinity of point $x_{s} \in(0,1)$.

The estimate of local domain of attraction is obtained in(Nefedov et al., 2013).

The aim of this work is to study the stationing of the solution to problem1 to the stationary stable solution. To do this, first of all, it is necessary to require the conditions for the existence of a front-type solution to problem1 formulated in(Antipov et al., 2014) and the conditions for the existence and stability of the stable stationary solution to problem formulated in(Vasil'eva, 1995). In order to formulate these conditions, we need to study the so-called associated systems for problems1 and2. 


\section{Associated systems}

For a detailed description of the solution behavior in the transition layer, we introduce stretched variables

$$
\xi=\frac{x-\hat{x}(t)}{\varepsilon}, \quad \xi_{s}=\frac{x-x_{s}}{\varepsilon} .
$$

The differential operators of equations1 and2 rewritten through stretched variables have the form:

$$
\begin{gathered}
\varepsilon \frac{\partial^{2}}{\partial x^{2}}-\frac{\partial}{\partial t}-A(\cdot, x) \frac{\partial}{\partial x}=\frac{1}{\varepsilon}\left(\frac{\partial^{2}}{\partial \xi^{2}}-\left(A(\cdot, \hat{x}(t)+\varepsilon \xi)-\frac{d \hat{x}}{d t}\right) \frac{\partial}{\partial \xi}+O(\varepsilon)\right), \\
\varepsilon \frac{d^{2}}{d x^{2}}-A(\cdot, x) \frac{d}{d x}=\frac{1}{\varepsilon}\left(\frac{d^{2}}{d \xi_{s}^{2}}-A\left(\cdot, x_{s}+\varepsilon \xi_{s}\right) \frac{d}{d \xi_{s}}+O(\varepsilon)\right) .
\end{gathered}
$$

We consider the so-called "associated equations"(Vasil'eva et al., 1995) for problems1 and2

$$
\frac{\partial^{2} \tilde{v}}{\partial \xi^{2}}=(A(\tilde{v}, x)-V) \frac{\partial \tilde{v}}{\partial \xi} \text { and } \frac{d^{2} \tilde{u}}{d \xi_{s}^{2}}=A(\tilde{u}, x) \frac{d \tilde{u}}{d \xi_{s}},
$$

where $x$ and $V$ are parameters. Note that $V=0$ in case of associated equation for problem2 so we shall further consider the first of these equations.

From the equivalent associated system

$$
\frac{\partial \tilde{v}}{\partial \xi}=\Phi, \quad \frac{\partial \Phi}{\partial \xi}=(A(\tilde{v}, x)-V) \Phi
$$

we come to the equation $\frac{\partial \Phi}{\partial \tilde{v}}=A(\tilde{v}, x)-V$ determining the trajectories on the phase plain $(\tilde{v}, \Phi)$ :

$$
\Phi^{(\mp)}(\tilde{v}, x, V)=\int_{\varphi^{(\mp)}(x)}^{\tilde{v}}(A(s, x)-V) d s, \quad \varphi^{(-)}(x)<\tilde{v}<\varphi^{(+)}(x) .
$$

Each of the points $\left(\varphi^{(\mp)}(x), 0\right)$ on the phase plane is a saddle rest point of system5. The phase trajectory $\Phi^{(-)}(\tilde{v}, x, V)$ enters the rest point $\left(\varphi^{(-)}(x), 0\right)$ when $\xi \rightarrow-\infty$, and $\Phi^{(+)}(\tilde{v}, x, V)$ enters the rest point $\left(\varphi^{(+)}(x), 0\right)$ when $\xi \rightarrow+\infty$. 
If at each instant of time there exists a pair of parameters $\left(x_{0}, V_{0}\right)$ for which the equality $\Phi^{(-)}\left(\tilde{v}, x_{0}, V_{0}\right)=$ $\Phi^{(+)}\left(\tilde{v}, x_{0}, V_{0}\right)$ holds the phase trajectories intersect and there exists the solution $\tilde{v}(\xi)$ to the equation 4 coming out the saddle point $\left(\varphi^{(-)}(x), 0\right)$ and entering the saddle point $\left(\varphi^{(+)}(x), 0\right)$. Moreover the parameters $x_{0}$ and $V_{0}$ are related by the equality

$$
V_{0}\left(x_{0}\right)=\left(\varphi^{(+)}\left(x_{0}\right)-\varphi^{(-)}\left(x_{0}\right)\right)^{-1} \int_{\varphi^{(-)}\left(x_{0}\right)}^{\varphi^{(+)}\left(x_{0}\right)} A\left(u, x_{0}\right) d u
$$

Let the Cauchy problem $\frac{d x_{0}}{d t}=\frac{\int_{\varphi(-)}^{\varphi^{(+)}\left(x_{0}\right)} A\left(u, x_{0}\right) d u}{\varphi^{(+)}\left(x_{0}\right)-\varphi^{(-)}\left(x_{0}\right)}, \quad x_{0}(0)=x_{00}$ has a unique solution $x_{0}(t) \in$ $(0 ; 1), \quad t>0$.

Let on the interval $(0 ; 1)$ there exists a unique solution $x_{s 0}$ of the equation $\int_{\varphi(-)}^{\varphi^{(+)}(x)} A(u, x) d u=0$.

Let the inequalities hold: $V_{0}>0$ if $x<x_{s 0}$ and $\frac{d V_{0}}{d x_{0}}\left(x_{s 0}\right)<0$.

\section{Study Algorithm}

We will conduct our research using the method of the upper and lower solutions(Kazdan \& Kramer, 1978; Wang, 1998). According to(Kazdan \& Kramer, 1978) if there exist the upper $(\beta(x, \varepsilon))$ and lower $(\alpha(x, \varepsilon))$ solutions of problem2 then there exists the solution $u_{\varepsilon}(x)$ to problem 2 enclosed between the upper and lower solutions: $\alpha(x, \varepsilon) \leq u_{\varepsilon}(x) \leq \beta(x, \varepsilon), \quad x \in[0,1]$.

According to(Wang, 1998) from the existence of the upper $(\hat{\beta}(x, t, \varepsilon))$ and lower $(\hat{\alpha}(x, t, \varepsilon))$ solutions of problem1 and from the inequality $\hat{\alpha}(x, 0, \varepsilon) \leq v_{\text {init }}(x, \varepsilon) \leq \hat{\beta}(x, 0, \varepsilon), \quad x \in[0,1]$ the existence of the solution $v_{\varepsilon}(x, t)$ to this problem follows for which the inequalities hold: $\hat{\alpha}(x, t, \varepsilon) \leq v_{\varepsilon}(x, t) \leq \hat{\beta}(x, t, \varepsilon), \quad x \in[0,1], t>$ 0 .

The upper and lower solutions of each of the problems 1 and 2 we will construct according to the asymptotic method of differential inequalities(Nefedov, 1995; Vasil'eva et al., 2010) as modifications of the second-order asymptotic approximations in the small parameter of these solutions, therefore we will denote them by the subscript "2".

According to(Nefedov et al., 2013) the domain $\left[\alpha_{2}(x, \varepsilon), \beta_{2}(x, \varepsilon)\right], x \in[0,1]$ is of the local domain of attraction of stable stationary solution.

In order to prove the stabilization theorem, we will show that during an asymptotically large but finite time interval $T$, the upper and lower solutions of problem 1 will be included in the domain of stability of the stationary solution, as a result of which the limit equality will follow: $\lim _{t \rightarrow+\infty}\left|v_{\varepsilon}(x, t)-u_{\varepsilon}(x)\right|=0$.

\section{Asymptotic approximation of moving front solution}

According to(Antipov et al., 2014) the $n-t h$ order asymptotic approximation $V_{n}(x, t, \varepsilon)$, of problem1 is constructed separately on each of the intervals $[0 ; \hat{x}]$ and $[\hat{x} ; 1]$ as a sum of two terms: $\bar{u}(x, \varepsilon)$, the regular 
part to that the solution is close away from $\hat{x}(t)$ vicinity and the transition layer function $Q(\xi, t, \varepsilon)$, where $\xi$ is the stretched variable3.

$$
\begin{gathered}
V_{n}(x, t, \varepsilon)= \\
\begin{cases}V_{n}^{(-)}(x, t, \varepsilon)=\bar{u}^{(-)}(x, \varepsilon)+Q^{(-)}(\xi, t, \hat{x}, V, \varepsilon), & (x, t) \in[0, \hat{x}] \times[0 ; T], \\
V_{n}^{(+)}(x, t, \varepsilon)=\bar{u}^{(+)}(x, \varepsilon)+Q^{(+)}(\xi, t, \hat{x}, V, \varepsilon), & (x, t) \in[\hat{x}, 1] \times[0 ; T] .\end{cases}
\end{gathered}
$$

The functions with superscript "(-)" are determined for $0 \leq x \leq \hat{x}(t)$ and $\xi \leq 0$ and the functions with superscript " $(+) "-$ for $\hat{x}(t) \leq x \leq 0$ and $\xi \geq 0$.

The functions $V_{n}^{(-)}$and $V_{n}^{+}$are continuously matched at a point $\hat{x}(t)$ at each instant of time so that the equality holds: $V_{n}^{(-)}(\hat{x}(t), t, \varepsilon)=V_{n}^{+}(\hat{x}(t), t, \varepsilon)=\frac{1}{2}\left(\varphi^{(-)}(\hat{x}(t))+\varphi^{(+)}(\hat{x}(t))\right)$. For the purposes of this study, we need a third-order asymptotic approximation; therefore, we use the following expansions of functions $\bar{u}^{(\mp)}(x, \varepsilon)$ and $Q^{(\mp)}(\xi, t, \hat{x}, V, \varepsilon)$ :

$$
V_{3}^{(\mp)}(\hat{x}(t), t, \varepsilon)=\sum_{i=0}^{3}\left(\varepsilon^{i} \bar{u}_{i}^{(\mp)}(x)+\varepsilon^{i} Q_{i}^{(\mp)}(\xi, t, \hat{x}, V)\right) .
$$

The main regular terms of the asymptotic approximation are determined by the Proposition: $\bar{u}_{0}^{(\mp)}(x, t)=$ $\varphi^{(\mp)}(x)$. The functions $\bar{u}_{i}^{(\mp)}(x)$ for $i \geq 1$ are the solutions to initial problems

$$
A\left(\varphi^{(\mp)}(x), x\right) \frac{d \bar{u}_{i}^{(\mp)}}{d x}=-W^{(\mp)}(x) \bar{u}_{i}^{(\mp)}+\bar{f}_{i}^{(\mp)}(x), \quad x \in(0,1), \bar{u}_{i}^{(-)}(0)=0, \bar{u}_{i}^{(+)}(1)=0
$$

with known functions $\bar{f}_{k}^{(\mp)}(x)$ and

$$
W^{(\mp)}(x)=A_{v}\left(\varphi^{(\mp)}(x), x\right) \frac{d \varphi^{(\mp)}}{d x}(x)+B_{v}\left(\varphi^{(\mp)}(x), x\right) .
$$

The 0 -th order transition layer functions $Q_{0}^{(\mp)}(\xi, t, \hat{x}, V)$ are the solutions to problems 
on half-line $\xi \leq 0$ for $Q_{0}^{(-)}(\xi, t, \hat{x}, V)$ and $\xi \geq 0$ for $Q_{0}^{(+)}(\xi, t, \hat{x}, V)$.

By use of the notation

$$
\tilde{v}(\xi, \hat{x}, V):=
$$

$\begin{cases}\varphi^{(-)}(\hat{x}(t))+Q_{0}^{(-)}(\xi, t, \hat{x}, V), & \xi \leq 0,0 \leq t \leq T \\ \varphi^{(+)}(\hat{x}(t))+Q_{0}^{(+)}(\xi, t, \hat{x}, V), & \xi \geq 0,0 \leq t \leq T\end{cases}$

(11)

equations?? take form4. According to section the there exist the functions

$$
\begin{gathered}
\Phi^{(-)}(\xi, \hat{x}, V)=\Phi^{(-)}(\tilde{v}(\xi), \hat{x}, V)=\frac{\partial \tilde{v}}{\partial \xi}(\xi, \hat{x}, V), \quad \xi \leq 0,0 \leq t \leq T, \\
\Phi^{(+)}(\xi, \hat{x}, V)=\Phi^{(+)}(\tilde{v}(\xi), \hat{x}, V)=\frac{\partial \tilde{v}}{\partial \xi}(\xi, \hat{x}, V), \quad \xi \geq 0,0 \leq t \leq T .
\end{gathered}
$$

For the transition layer the following standard estimates hold:

$$
\left|Q_{0}^{(\mp)}(\xi, t, \hat{x}, V)\right| \leq C_{0} e^{-\kappa|\xi|}
$$

where $C_{0}$ and $\kappa$ are some positive constants. The analogous estimates hold for the derivatives of functions $Q^{(\mp)}(\xi, t, \hat{x}, V)$.

Next, for brevity, we introduce the notations

$$
\tilde{A}(\xi, t)=A(\tilde{v}(\xi, \hat{x}(t)), \hat{x}(t)), \quad \tilde{B}(\xi, t)=B(\tilde{v}(\xi, \hat{x}(t)), \hat{x}(t))
$$

and the same notations for the derivatives of functions $A(\tilde{v}(\xi, \hat{x}(t)), \hat{x}(t))$ and $B(\tilde{v}(\xi, \hat{x}(t)), \hat{x}(t))$. The $i-t h$ order transition layer functions, $i=1,2,3$ are the solutions to linear problems 
where $f_{i}^{(\mp)}(\xi, t)$ are known functions depending on further determined terms of asymptotic approximation with $j<i$, in particular

$f_{1}^{(\mp)}(\xi, t)=\Phi^{(\mp)}(\xi, \hat{x}, V)\left(\frac{\partial \tilde{A}}{\partial u}(\xi, t)\left(\bar{u}_{1}^{(\mp)}(\hat{x}(t))+\frac{d \varphi^{(\mp)}}{d x}(\hat{x}(t)) \cdot \xi\right)+\frac{\partial \tilde{A}}{\partial x}(\xi, t) \cdot \xi\right)+\tilde{A}(\xi, t) \frac{d \varphi^{(\mp)}}{d x}(\hat{x}(t))+\tilde{B}(\xi, t)+\frac{\partial Q_{0}^{(\mp)}}{\partial t}$

Problems?? can be solved explicitly:

$$
Q_{i}^{(\mp)}(\xi, t, \hat{x}, V)=-\bar{u}_{i}^{(\mp)}(\hat{x}(t)) \frac{\Phi^{(\mp)}(\xi, \hat{x}, V)}{\Phi^{(\mp)}(0, \hat{x}, V)}+\Phi^{(\mp)}(\xi, \hat{x}, V) \int_{0}^{\xi} \frac{d s}{\Phi^{(\mp)}(s, \hat{x}, V)} \int_{\mp \infty}^{s} f_{i}^{(\mp)}(\eta, t) d \eta
$$

For functions $Q_{i}^{(\mp)}(\xi, t, \hat{x}, V), i=1,2$ the estimates similar to13 hold.

Functions $V_{3}$ is continuous due to the boundary conditions at $\xi=0$ of problems?? and ??.

We also represent the functions $\hat{x}(t)$ and $V(t)=d \hat{x} / d t$ as expansions

$$
\hat{x}(t)=x_{0}(t)+\varepsilon x_{1}(t)+\varepsilon^{2} x_{2}(t)+\ldots, \quad V(t)=\frac{d x_{0}}{d t}+\varepsilon \frac{d x_{1}}{d t}+\varepsilon^{2} \frac{d x_{2}}{d t}+\ldots
$$

The coefficients $x_{i}(t)$ and $V_{i}(t)$ will be determined from the following conditions for the derivatives:

Taking expansions 17 into account we obtain that $x_{0}(t)$ is the function from Proposition (see notations11 and 12 ) and the functions $x_{i}(t), i=1,2$ are the solutions to linear problems 
where $G_{i}(t)$ are known functions, in particular

$G_{1}(t)=\left(\varphi^{(-)}\left(x_{0}(t)\right)-\varphi^{(+)}\left(x_{0}(t)\right)\right)^{-1}\left(\frac{d \varphi^{(-)}}{d x}\left(x_{0}(t)\right)-\frac{d \varphi^{(+)}}{d x}\left(x_{0}(t)\right)+\frac{\partial Q_{1}^{(-)}}{\partial \xi}\left(0, t, x_{0}, V_{0}\left(x_{0}\right)\right)-\frac{\partial Q_{1}^{(+)}}{\partial \xi}\left(0, t, x_{0}, V_{0}\left(x_{0}\right)\right)\right)$

\section{The moving front upper and lower solutions.}

In(Antipov et al., 2014) the following upper and lower solutions of problem1 are constructed:

$$
\hat{\beta}_{2}(x, t, \bar{x}, \bar{V}, \varepsilon)=
$$

$\left\{\begin{array}{ll}\hat{\beta}_{2}^{(-)}(x, t, \bar{x}, \bar{V}, \varepsilon), & 0 \leq x \leq \bar{x}(t), t>0, \\ \hat{\beta}_{2}^{(+)}(x, t, \bar{x}, \bar{V}, \varepsilon), & \bar{x}(t) \leq x \leq 1, t>0 ;\end{array} \quad 2(x, t, \underline{x}, \underline{V}, \varepsilon)=\right.$

$\begin{cases}\hat{\alpha}_{2}^{(-)}(x, t, \underline{x}, \underline{V}, \varepsilon), \quad 0 \leq x \leq \underline{x}(t), t>0 \\ \hat{\alpha}_{2}^{(+)}(x, t, \underline{x}, \underline{V}, \varepsilon), \quad \underline{x}(t) \leq x \leq 1, t>0\end{cases}$

where

$$
\begin{aligned}
& \hat{\beta}_{2}^{(\mp)}(x, t, \bar{x}, \bar{V}, \varepsilon)=V_{3}^{(\mp)}(x, t, \varepsilon)+\varepsilon^{2}\left(\mu^{(\mp)}(x)+q_{0}^{(\mp)}(\bar{\xi}, \bar{x}, \bar{V})+\varepsilon q_{1}^{(\mp)}(\bar{\xi}, \bar{x}, \bar{V})\right), \\
& \hat{\alpha}_{2}^{(\mp)}(x, t, \underline{x}, \underline{V}, \varepsilon)=V_{3}^{(\mp)}(x, t, \varepsilon)-\varepsilon^{2}\left(\mu^{(\mp)}(x)+q_{0}^{(\mp)}(\underline{\xi}, \underline{x}, \underline{V})+\varepsilon q_{1}^{(\mp)}(\underline{\xi}, \underline{x}, \underline{V})\right),
\end{aligned}
$$

$\bar{x}=X_{2}(t, \varepsilon)-\varepsilon^{2} \delta(t), \underline{x}=X_{2}(t, \varepsilon)+\varepsilon^{2} \delta(t), X_{2}(t, \varepsilon):=x_{0}(t)+\varepsilon x_{1}(t)+\varepsilon^{2} x_{2}(t) \bar{V}=d \bar{x} / d t, \bar{\xi}=(x-\bar{x}) / \varepsilon$, $\underline{V}=d \underline{x} / d t, \underline{\xi}=(x-\underline{x}) / \varepsilon$.

The functions $\mu^{(\mp)}(x)$ are the solutions to problems

$$
\frac{d \mu^{(\mp)}}{d x}+W^{(\mp)}(x) \mu^{(\mp)}(x)=R \cdot\left(A\left(\varphi^{(\mp)}(x), x\right)\right)^{-1}, \mu^{(-)}(0)=R^{(-)}, \quad \mu^{(+)}(1)=R^{(+)},
$$

where $R, R^{(-)}, R^{(+)}$are positive constants, and $W^{(\mp)}(x)$ is notation10.

The explicit expressions for $\mu^{(\mp)}(x)$ are the following: 
In consequence of Proposition the functions $\mu^{(\mp)}(x)$ take positive values when $x \in[0 ; 1]$.

The functions $q_{i}^{(\mp)}(\bar{\xi}, \bar{x}, \bar{V}), i=0,1$ included in the upper solution are determined as the solutions to problems

We set the problems for functions with superset "(-)" on half-line $\bar{\xi} \leq 0$, and with superset " $(+)$ " on half-line $\bar{\xi} \geq 0$. The functions $q_{i} F\left(\xi, \mu^{(\mp)}(\bar{x}(t))\right)$ are known and have exponential estimates like13.

We set the same problems to determine the low solution terms $\left.q_{i}^{(\mp)}(\underline{\xi}, \underline{x}, \underline{V})\right), i=0,1$, changing $\bar{\xi}$ by $\underline{\xi}$ and $\bar{x}$ by $\underline{x}$.

The functions $q_{i}^{(\mp)}(\bar{\xi}, \bar{x}, \bar{V})$ and $q_{i}^{(\mp)}(\underline{\xi}, \underline{x}, \underline{V} i=0,1$ decrease exponentially and have estimates like13.

The function $\delta(t)$ in the expressions for $\bar{x}$ and $\underline{x}$ is determined as a solution to initial problem

$$
\frac{d \delta}{d t}=\frac{d V_{0}}{d x_{0}}\left(x_{0}(t)\right) \cdot \delta+F(t), \quad t \in(0 ; T], \quad \delta(0)=\delta^{0},
$$

where $F(t)$ is a known function that is bounded and strictly positive (see(Antipov et al., 2014)) and $\delta^{0}>0$. The solution $\delta(t)$ is also bounded and strictly positive for $t \geq 0$.

\section{Stationary solution asymptotic approximation}

The asymptotic approximation $U_{3}(x, \varepsilon)$, of promlem2 solution is constructed in(Vasil'eva, 1995) in a form

$$
U_{3}(x, \varepsilon)=
$$

$\begin{cases}U_{3}^{(-)}=\bar{u}^{(-)}(x, \varepsilon)+Q_{s}^{(-)}\left(\xi_{s}, x_{s}, \varepsilon\right), & x \in\left[0, x_{s}\right], \\ U_{3}^{(+)}=\bar{u}^{(+)}(x, \varepsilon)+Q_{s}^{(+)}\left(\xi_{s}, x_{s}, \varepsilon\right), & x \in\left[x_{s}, 1\right]\end{cases}$

where $\bar{u}^{(\mp)}(x, \varepsilon)$ is regular part similar to one in expression8 and $Q_{s}^{(\mp)}\left(\xi_{s}, x_{s}, \varepsilon\right)$ are transition layer functions depending on variable $\xi_{s}$ (see 3 ).

We represent function $U_{3}$ as expansion on $\varepsilon$ exponents similar to expansion9.

The functions $Q_{s 0}^{(\mp)}\left(\xi_{s}, x_{s}\right)$ are the solutions to problems 
$\frac{d^{2} Q_{s 0}^{(\mp)}}{d \xi_{s}^{2}}-A\left(\varphi^{(\mp)}\left(x_{s}\right)+Q_{s 0}^{(\mp)}, x_{s}\right) \frac{d Q_{s 0}^{(\mp)}}{d \xi_{s}}=0 ; \quad Q_{s 0}^{(\mp)}\left(0, x_{s}\right)+\varphi^{(\mp)}\left(x_{s}\right)=\frac{1}{2}\left(\varphi^{(-)}\left(x_{s}\right)+\varphi^{(-)}\left(x_{s}\right)\right), \quad Q_{0}^{(\mp)}\left(\mp \infty, x_{s}\right)=0$

on half-line $\xi_{s} \leq 0$ for $Q_{s 0}^{(-)}\left(\xi_{s}, x_{s}\right)$ and on half-line $\xi_{s} \geq 0$ for $Q_{0}^{(+)}\left(\xi_{s}, x_{s}\right)$ and the exponential estimates of the same form as13 where $\xi$ is changed by $\xi_{s}$ hold.

Let's introduce the notations

$$
\tilde{u}\left(\xi_{s}, x_{s}\right):=
$$

$\begin{cases}\varphi^{(-)}\left(x_{s}\right)+Q_{s 0}^{(-)}\left(\xi_{s}, x_{s}\right), & \xi_{s} \leq 0, \\ \varphi^{(+)}\left(x_{s}\right)+Q_{s 0}^{(+)}\left(\xi_{s}, x_{s}\right), & \xi_{s} \geq 0 .\end{cases}$

$\begin{cases}\Phi^{(-)}\left(\xi_{s}, x_{s}, 0\right):=\Phi^{(-)}\left(\tilde{u}\left(\xi_{s}, x_{s}\right), x_{s}, 0\right)=\frac{d \tilde{u}}{d \xi_{s}}\left(\xi_{s}, x_{s}\right), & \xi_{s} \leq 0, \\ \Phi^{(+)}\left(\xi_{s}, x_{s}, 0\right):=\Phi^{(+)}\left(\tilde{u}\left(\xi_{s}, x_{s}\right), x_{s}, 0\right)=\frac{d \tilde{u}}{d \xi_{s}}\left(\xi_{s}, x_{s}\right), & \xi_{s} \geq 0 .\end{cases}$

The existence of functions $\Phi^{(\mp)}\left(\xi_{s}, x_{s}, 0\right)$ follows from conclusions of section.

For brief we will further use notations $\tilde{A}\left(\xi_{s}\right)=A\left(\tilde{u}\left(\xi_{s}, x_{s}\right), x_{s}\right), \quad \tilde{B}\left(\xi_{s}\right)=B\left(\tilde{u}\left(\xi_{s}, x_{s}\right), x_{s}\right)$. and the same for derivatives of functions $A\left(\tilde{u}\left(\xi_{s}, x_{s}\right), x_{s}\right.$ and $B\left(\tilde{u}\left(\xi_{s}, x_{s}\right), x_{s}\right.$.

The $i$-th order transition layer functions $(i \geq 1)$ we determine as solutions to linear problems

$* \frac{d^{2} Q_{s i}^{(\mp)}}{d \xi_{s}^{2}}-\tilde{A}\left(\xi_{s}\right) \frac{d Q_{s i}^{(\mp)}}{d \xi_{s}}-\frac{\partial \tilde{A}}{\partial u}\left(\xi_{s}\right) \Phi^{(\mp)}\left(\xi_{s}, x_{s}, 0\right) Q_{s i}^{(\mp)}=f_{s i}^{(\mp)}\left(\xi_{s}\right), \quad Q_{s i}^{(\mp)}\left(0, x_{s}\right)=-\bar{u}_{i}^{(\mp)}\left(x_{s}\right), \quad Q_{i}^{(\mp)}\left(\mp \infty, x_{s}\right)=0$

with known functions $f_{s k}^{(\mp)}\left(\xi_{s}\right)$, in particular

$f_{s 1}^{(\mp)}\left(\xi_{s}\right)=\Phi^{(\mp)}\left(\xi_{s}, x_{s}, 0\right)\left(\frac{\partial \tilde{A}}{\partial u}\left(\xi_{s}\right)\left(\bar{u}_{1}^{(\mp)}\left(x_{s}\right)+\frac{d \varphi^{(\mp)}}{d x}\left(x_{s}\right) \cdot \xi_{s}\right)+\frac{\partial \tilde{A}}{\partial x}\left(\xi_{s}\right) \cdot \xi_{s}\right)+\tilde{A}\left(\xi_{s}\right) \frac{d \varphi^{(\mp)}}{d x}\left(x_{s}\right)+\tilde{B}\left(\xi_{s}\right)$.

The explicit solutions to these problems are

$$
Q_{s i}^{(\mp)}\left(\xi_{s}, x_{s}\right)=-\bar{u}_{i}^{(\mp)}\left(x_{s}\right) \frac{\Phi^{(\mp)}\left(\xi_{s}, x_{s}, 0\right)}{\Phi^{(\mp)}\left(0, x_{s}, 0\right)}+\Phi^{(\mp)}\left(\xi_{s}, x_{s}, 0\right) \int_{0}^{\xi_{s}} \frac{d s}{\Phi^{(\mp)}\left(s, x_{s}, 0\right)} \int_{\mp \infty}^{s} f_{i}^{(\mp)}(\eta, t) d \eta .
$$


For the functions $Q_{s i}^{(\mp)}\left(\xi_{s}, x_{s}\right)$ the exponential estimates are also valid.

We represent the value $x_{s}$ as an expansion in $\varepsilon$ exponents $x_{s}=x_{s 0}+\varepsilon x_{s 1}+\varepsilon^{2} x_{s 2}+\ldots$, where coefficients $x_{s i}, i=0,1,2$ we determine from the condition for derivatives similar to??

The coefficient $x_{s 0}$, is determined by Proposition and the $x_{s i}, i=1,2$ are the solutions of equations

$$
-\frac{d V_{0}}{d x}\left(x_{s 0}, 0\right) x_{s i}=G_{s i},
$$

where $G_{s i}$ are known values, in particular

$$
G_{s 1}=\left(\varphi^{(-)}\left(x_{0 s}\right)-\varphi^{(+)}\left(x_{0 s}\right)\right)^{-1}\left(\frac{d \varphi^{(-)}}{d x}\left(x_{s 0}\right)-\frac{d \varphi^{(+)}}{d x}\left(x_{s 0}\right)+\frac{\partial Q_{s 1}^{(-)}}{\partial \xi_{s}}\left(0, x_{s 0}\right)-\frac{\partial Q_{s 1}^{(+)}}{\partial \xi_{s}}\left(0, x_{s 0}\right)\right) .
$$

Equations25 are solvable due to Proposition.

\section{The upper and lower solutions of the stationary problem}

The upper and lower solutions of problem2 have the form(Nefedov et al., 2013):

$$
\beta_{2}\left(x, \bar{x}_{s}, \varepsilon\right)=
$$

$\begin{cases}\beta_{2}^{(-)}\left(x, \bar{x}_{s}, \varepsilon\right), & 0 \leq x \leq \bar{x}_{s}, \quad \alpha_{2}\left(x, \underline{x}_{s}, \varepsilon\right)= \\ \beta_{2}^{(+)}\left(x, \bar{x}_{s}, \varepsilon\right), & \bar{x}_{s} \leq x \leq 1 ;\end{cases}$

$\begin{cases}\alpha_{2}^{(-)}\left(x, \underline{x}_{s}, \varepsilon\right), & 0 \leq x \leq \underline{x}_{s}, \\ \alpha_{2}^{(+)}\left(x, \underline{x}_{s}, \varepsilon\right), & \underline{x}_{s} \leq x \leq 1,\end{cases}$

where

$$
\begin{aligned}
& \beta_{2}^{(\mp)}\left(x, \bar{x}_{s}, \varepsilon\right)=U_{3}^{(\mp)}(x, \varepsilon)+\varepsilon^{2}\left(\mu_{s}^{(\mp)}(x)+q_{s 0}^{(\mp)}\left(\bar{\xi}_{s}, \bar{x}_{s}\right)+\varepsilon q_{s 1}^{(\mp)}\left(\bar{\xi}_{s}, \bar{x}_{s}\right)\right), \\
& \alpha_{2}^{(\mp)}\left(x, \underline{x}_{s}, \varepsilon\right)=U_{3}^{(\mp)}(x, \varepsilon)-\varepsilon^{2}\left(\mu_{s}^{(\mp)}(x)+q_{s 0}^{(\mp)}\left(\underline{\xi}_{s}, \underline{x}_{s}\right)+\varepsilon q_{s 1}^{(\mp)}\left(\underline{\xi}_{s}, \underline{x}_{s}\right)\right) .
\end{aligned}
$$


Here $\bar{x}_{s}=X_{s 2}(\varepsilon)-\varepsilon^{2} \delta_{s}, \underline{x}=X_{s 2}(\varepsilon)+\varepsilon^{2} \delta_{s}, X_{s 2}(\varepsilon):=x_{s 0}+\varepsilon x_{s 1}+\varepsilon^{2} x_{s 2}, \bar{\xi}_{s}=\left(x-\bar{x}_{s}\right) / \varepsilon, \underline{\xi}_{s}=\left(x-\underline{x}_{s}\right) / \varepsilon$, functions $\mu_{s}^{(\mp)}(x)$ are given by expressions ?? the terms $q_{s i}^{(\mp)} i=0,1$ have the similar sense as analogous terms in19 and are determined from the same problems as?? with $V=0$ and $\bar{x}_{s}$ instead of $\bar{x}(t)$ (for the upper solution) and $\underline{x}_{s}$ instead of $\underline{x}(t)$ for the lower.

The value $\delta_{s}$ in expressions $\bar{x}_{s}$ and $\underline{x}_{s}$ is determined from equation

$$
-\frac{d V_{0}}{d x}\left(x_{s 0}\right) \cdot \delta_{s}=F_{s}
$$

where $F_{s}$ is known positive value. Due to Proposition the value $\delta_{s}$ is positive.

\section{Large time estimates}

We denote

$$
\Delta(t):=\bar{x}_{s}-\bar{x}(t)=\Delta_{0}(t)+\varepsilon \Delta_{1}(t)+\varepsilon^{2} \Delta_{2}(t)+\varepsilon^{2}\left(\delta(t)-\delta_{s}\right), \text { where } \Delta_{i}(t):=x_{s i}-x_{i}(t) .
$$

Here the function $\delta(t)$ is determined from Cauchy problem21 and the value $\delta_{s}$ from equation27.

The parameter $\bar{V}$ has the following representation:

$$
\bar{V}=V_{0}\left(x_{0}\right)+\varepsilon \bar{V}_{1}\left(x_{0}\right)+\varepsilon^{2} \bar{V}_{2}\left(x_{0}\right)-\varepsilon^{2} \frac{d \delta}{d t}(t)
$$

where $V_{0}$ is determined by expression $7, V_{i}\left(x_{0}\right)=\frac{d x_{i}}{d t}$, and for $x_{i}(t), i=1,2$ we have set problems??

\section{The estimate for the function $\Delta_{0}(t)$.}

The functions $V_{i}\left(x_{0}\right), i=0,1$ are of the same smoothness as $A(u, x), B(u, x)$, and $\varphi^{(\mp)}(x)$. Besides $V_{0}\left(x_{s 0}\right)=$ 0 in consequence of Propositions and and the equality $V_{1}\left(x_{s 0}\right)=0$ follows from equations ??, 25. From that we get the following expression for $\bar{V}$ :

$$
\bar{V}=\left(\frac{d V_{0}}{d x_{0}}\left(x_{s 0}\right)+\varepsilon \frac{d V_{1}}{d x_{0}}\left(x_{s 0}\right)\right) \Delta_{0}(t)+O\left(\Delta_{0}^{2}(t)+O\left(\varepsilon^{2}\right)\right) .
$$


Let's rewrite Cauchy problem mentioned in Proposition taking into account expression 7 and definition (28) in a form

$$
\frac{d \Delta_{0}}{d t}=-V_{0}\left(x_{s 0}-\Delta_{0}(t)\right), \quad \Delta_{0}(0)=x_{s 0}-x_{00}
$$

By virtue of Proposition and the inequality $d V_{0} / d \Delta_{0}<0$ consequent from Proposition the point $\Delta_{0}=0$ is asymptotically stable rest point of this equation thus the estimate is valid:

$$
0 \leq \Delta_{0}(t) \leq P_{0} \exp \left\{\frac{d V_{0}}{d x_{0}}\left(x_{s 0}\right) t\right\}
$$

where $P_{0}$ is a positive constant.

\section{The estimates for function $Q_{i}^{(+)}(\bar{\xi}, t, \bar{x}, \bar{V}) i=0,1$}

The function $Q_{0}^{(+)}(\bar{\xi}, t, \bar{x}, \bar{V})$ is the solution to Cauchy problem

$$
\frac{\partial Q_{0}^{(+)}}{\partial \bar{\xi}}=\Phi^{(+)}(\bar{\xi}, \bar{x}(t), \bar{V}), \quad Q_{0}^{(+)}(0, t, \bar{x}, \bar{V})=\frac{1}{2}\left(\varphi^{(-)}(\bar{x}(t))-\varphi^{(+)}(\bar{x}(t))\right),
$$

with the functions $\Phi(\bar{\xi}, \bar{x}(t), \bar{V})$ determined by expressions 6 where $\xi$ is replaced by $\bar{\xi}, \hat{x}$ by $\bar{x}, V$ by $\bar{V}$.(see5, notations 11,12) and the boundary condition at $\xi=0$ of problem ??)

The functions $Q_{s 0}^{(+)}\left(\bar{\xi}_{s}, \bar{x}_{s}\right)$ are the solutions to Cauchy problems (see22):

$$
\frac{\partial Q_{s 0}^{(+)}}{\partial \bar{\xi}_{s}}=\Phi^{(+)}\left(\bar{\xi}_{s}, \bar{x}_{s}, 0\right), \quad Q_{s 0}^{(+)}\left(0, \bar{x}_{s}\right)=\frac{1}{2}\left(\varphi^{(-)}\left(\bar{x}_{s}\right)-\varphi^{(+)}\left(\bar{x}_{s}\right)\right) .
$$

From the known theorem on the dependence of the Cauchy problem solution on a parameter, taking into account expressions29 and $\Delta_{0}(t)=\Delta(t)+O(\varepsilon)$ (see28) we come to the equality

$$
Q_{0}^{(+)}(\bar{\xi}, t, \bar{x}, \bar{V})=Q_{s 0}^{(+)}\left(\bar{\xi}, \bar{x}_{s}\right)+\frac{\partial Q_{0}^{(+)}}{\partial V}\left(\bar{\xi}, t, x^{*}, V^{*}\right) \bar{V}+\frac{\partial Q_{0}^{(+)}}{\partial \bar{x}}\left(\bar{\xi}, t, x^{*}, V^{*}\right) \Delta(t)
$$

where $x^{*}=\bar{x}_{s}-\theta_{1} \Delta(t), V^{*}=\theta_{2} \bar{V}, 0<\theta_{1,2}<1$. 
Based on the form of equations ?? we come to the conclusion that for derivatives $\frac{\partial Q_{0}^{(+)}}{\partial V}\left(\bar{\xi}, t, x^{*}, V^{*}\right)$ and $\frac{\partial Q_{0}^{(+)}}{\partial \bar{x}}\left(\bar{\xi}, t, x^{*}, V^{*}\right)$ the exponential estimates similar to 13 hold. From that and the expressions 29 , and $\Delta_{0}(t)=\Delta(t)+O(\varepsilon)$ for small $\Delta_{0}(t)$ (sufficiently large $t$ ) we obtain the estimate

$$
Q_{0}^{(+)}(\bar{\xi}, t, \bar{x}, \bar{V})=Q_{s 0}^{(+)}\left(\bar{\xi}, \bar{x}_{s}\right)+\chi_{0}(\bar{\xi})\left(\Delta(t)+O\left(\varepsilon \Delta(t)+\Delta^{2}(t)+\varepsilon^{2}\right)\right),
$$

where the function $\chi_{0}(\bar{\xi})$ has the estimate like13,

From the explicit expressions 16 and24 for functions $Q_{1}^{(\mp)}(\bar{\xi}, t, \bar{x}, \bar{V})$ and $Q_{s 1}^{(\mp)}\left(\bar{\xi}, \bar{x}_{s}\right)$ taking into account expressions 15, 23 and 6 and also the estimates 29 and 31 we obtain the expression

$$
Q_{1}^{(+)}(\bar{\xi}, t, \bar{x}, \bar{V})=Q_{s 1}^{(+)}\left(\bar{\xi}, \bar{x}_{s}\right)+\chi_{1}(\bar{\xi})\left(\Delta(t)+O\left(\varepsilon \Delta(t)+\Delta^{2}(t)+\varepsilon^{2}\right)\right)
$$

where function $\chi_{1}(\bar{\xi})$ has the estimate like13,

\subsection{The estimate for $\Delta_{1}(t)$.}

Let's obtain the estimate for function $\Delta_{1}(t)$ (see 28). Based on equations?? and25 with $i=1$, taking into account explicit expressions 18 and26 and also expression29, we come to the following problem for function $\Delta_{1}(t)$ :

$$
\frac{d \Delta_{1}}{d t}=\frac{d V_{0}}{d x_{0}}\left(x_{s 0}\right) \Delta_{1}+F_{1}(t) \Delta_{0}(t), \quad \Delta_{1}(0)=x_{s 1}, \quad \text { where } F_{1}(t)=-\frac{d^{2} V_{0}}{d x_{0}^{2}}\left(x_{1}^{*}\right) x_{1}(t)-\frac{\partial G_{1}}{\partial x_{0}}\left(x_{2}^{*}\right),
$$

$x_{1,2}^{*}=x_{0}(t)+\theta_{1,2}(t) \Delta_{0}(t), 0<\theta_{1,2}(t)<1$.

By using estimate30 we get

$$
\left|\Delta_{1}(t)\right| \leq P_{1} \exp \left\{\frac{d V_{0}}{d x_{0}}\left(x_{s 0}\right) t\right\}
$$

where $P_{1}>0$. 


\section{the estimates for functions of the 2-nd and 3-d order.}

From the estimates $31,32,30$ and 33 for the functions $Q_{0}^{(\mp)}, Q_{1}^{(\mp)}, \Delta_{0}, \Delta_{1}$, that are included in the right-hand side of equation (??) for $i=2$, we obtain the estimates for the functions $Q_{2}^{(\mp)}(\bar{\xi}, t, \bar{x}, \bar{V})$ and $q_{0}^{(\mp)}(\bar{\xi}, \bar{x}, \bar{V})$ analogous to (32), and for the function $\Delta_{2}(t)$ the estimate analogous to33. Using estimates of second-order functions, we obtain an estimate for the function $\delta(t)$ similar to 33 and then by induction the estimates for the 3-d order functions $Q_{3}^{(\mp)}(\bar{\xi}, t, \bar{x}, \bar{V})$ and $q_{1}^{(\mp)}(\bar{\xi}, \bar{x}, \bar{V})$.

\section{The main result}

For every Holder continuous function $v_{\text {init }}(x, \varepsilon)$, in settlement 1 , that is enclosed between the upper $\hat{\beta}_{2}(x, t, \bar{x}(0),(d \bar{x} / d t)(0), \varepsilon)$ solution of problem1 and lower $\alpha_{2}\left(x, \underline{x}_{s}, \varepsilon\right)$ solution of stationary problem2, the limiting equality holds:

$$
\lim _{t \rightarrow \infty}\left|v_{\varepsilon}(x, t)-u_{\varepsilon}(x)\right|=0, \quad x \in[0,1] .
$$

The proof of the theorem consists of the two stages:

Stage 1$)$. We prove that the functions $\hat{\beta}_{2}(x, t, \bar{x}, \bar{V}, \varepsilon)$ and $\alpha_{2}\left(x, \underline{x}_{s}, \varepsilon\right)$ are the ordered upper and lower solutions of problem 1. Then according to the theorem proved in(Wang, 1998) mentioning the analogous theorem from(Pao, 1992) the solution of the problem1 at any time will be enclosed between these upper and lower solutions.

Stage 2 ). We prove that for sufficiently large $t>T$ the inequality holds

$$
\hat{\beta}_{2}(x, t, \bar{x}, \bar{V}, \varepsilon)<\beta_{2}\left(x, \bar{x}_{s}, \varepsilon\right) .
$$

This will mean that the solution of the problem1 for sufficiently large $t$ will fall inside the local stability domain of the stationary problem2 solution.

The proof of stage 1). As the function $\alpha_{2}\left(x, \underline{x}_{s}, \varepsilon\right)$ is the lower solution of problem 2 and doesn't depend on time then it is also the lower solution to problem1 (see. (Kazdan \& Kramer, 1978; Wang, 1998)). The ordering of the upper and lower solutions of parabolic problem follows from the theorem proved in(Pao, 1992).

The proof of stage 2). From the estimates30 and33 it follows that at the moment of time $T=$ $2\left|\frac{d V_{0}}{d x_{0}}\left(x_{s 0}\right)\right|^{-1}|\ln \varepsilon|$, it holds $\Delta_{0}=O\left(\varepsilon^{2}\right), \delta(t)=O\left(\varepsilon^{2}\right)$, then

$$
\bar{x}(t)-\bar{x}_{s}=\varepsilon^{2}\left(\delta_{s}-\Delta_{0}\right)+o\left(\varepsilon^{2}\right),
$$

where $\delta_{s}>0$ is determined from equation 27 and it can be chosen sufficiently large that $\delta_{s}-\Delta_{0}>0$. 
We will prove that relation34 holds separately for each of the segments: $0<x<\bar{x}_{s}, \bar{x}_{s} \leq x \leq \hat{x}(t)$, $\hat{x}(t)<x<1$.

Proof on the segment $\bar{x}_{s} \leq x \leq \bar{x}(t)$.

On this segment the equality holds $\bar{\xi}_{s}-\bar{\xi}=\varepsilon\left(\delta_{s}-\Delta_{0}\right)+o(\varepsilon)$. For the difference between the upper solutions of problems 2 and 1 on this segment by use of expression 31 , and 35 we can write

$$
\begin{gathered}
\beta_{2}^{(+)}\left(x, \bar{x}_{s}, \varepsilon\right)-\hat{\beta}_{2}^{(-)}(x, t, \bar{x}, \bar{V}, \varepsilon)=\varphi^{(+)}(x)+Q_{s 0}^{(+)}\left(\bar{\xi}_{s}, \bar{x}_{s}\right)-\varphi^{(-)}(x)-Q_{0}^{(-)}(\bar{\xi}, t, \bar{x}, \bar{V})+O\left(\varepsilon^{2}\right)= \\
=\varphi^{(+)}\left(\bar{x}_{s}\right)+Q_{s 0}^{(+)}\left(\bar{\xi}_{s}, \bar{x}_{s}\right)-\varphi^{(-)}\left(\bar{x}_{s}\right)-Q_{s 0}^{(-)}\left(\bar{\xi}, \bar{x}_{s}\right)+O\left(\varepsilon^{2}\right)=\Phi\left(0, \bar{x}_{s}, 0\right)\left(\bar{\xi}_{s}-\bar{\xi}\right)+O\left(\varepsilon^{2}\right)=\varepsilon \Phi\left(0, \bar{x}_{s}, 0\right)\left(\delta_{s}-\Delta_{0}\right)+O\left(\varepsilon^{2}\right.
\end{gathered}
$$

where the term $\Phi\left(0, \bar{x}_{s}, 0\right):=\Phi^{(-)}\left(0, \bar{x}_{s}, 0\right)=\Phi^{(+)}\left(0, \bar{x}_{s}, 0\right)$ ) is positive as the function $\tilde{u}$ increases (see22 and Proposition) and so the right -hand side is positive via the choice of $\delta_{s}$

Proof on the segment $\bar{x}(t) \leq x \leq 1$.

For the difference between the upper solutions of problems 2 and 1 on this segment we have the expression

$$
\begin{gathered}
\beta_{2}^{(+)}\left(x, \bar{x}_{s}, \varepsilon\right)-\hat{\beta}_{2}^{(+)}(x, t, \bar{x}, \bar{V}, \varepsilon)= \\
Q_{s 0}^{(+)}\left(\bar{\xi}_{s}, \bar{x}_{s}\right)-Q_{0}^{(+)}(\bar{\xi}, t, \bar{x}, \bar{V})+\varepsilon\left(Q_{s 1}^{(+)}\left(\bar{\xi}_{s}, \bar{x}_{s}\right)-Q_{1}^{(+)}(\bar{\xi}, t, \bar{x}, \bar{V})\right)+\varepsilon^{2}\left(Q_{s 2}^{(+)}\left(\bar{\xi}_{s}, \bar{x}_{s}\right)-Q_{2}^{(+)}(\bar{\xi}, t, \bar{x}, \bar{V})\right)+ \\
+\varepsilon^{2}\left(\mu_{s}(x)-\mu(x)+q_{s 0}^{(+)}\left(\bar{\xi}_{s}, \bar{x}_{s}\right)-q_{0}^{(+)}(\bar{\xi}, \bar{x}, \bar{V})\right)+\varepsilon^{3}\left(Q_{s 3}^{(+)}\left(\bar{\xi}_{s}, \bar{x}_{s}-Q_{3}^{(+)}(\bar{\xi}, t, \bar{x}, \bar{V})\right)\right)+\varepsilon^{3}\left(q_{s 1}^{(+)}\left(\bar{\xi}_{s}, \bar{x}_{s}\right)-q_{1}^{(+)}(\bar{\xi}, \bar{x}, \bar{V})\right)
\end{gathered}
$$

We choose the values $R_{0}^{(\mp)}$ and $R$ in problem statements20 for functions $\mu^{(\mp)}(x)$ and $\mu_{s}^{(\mp)}(x)$ in a way that for $x \in[0,1]$ the inequalities hold $\mu_{s}^{(\mp)}(x)=2 \mu^{(\mp)}(x)$.

For the mentioned value $T$, taking into account expressions 31,32 and the choice of functions $\mu^{(\mp)}(x)$ and $\mu_{s}^{(\mp)}(x)$ the proof of the inequality

$$
\beta\left(x, \bar{x}_{s}, \varepsilon\right)-\hat{\beta}_{2}(x, t, \bar{x}, \bar{V}, \varepsilon) \geq 0, \quad, 0 \leq x \leq 1, t \geq T
$$

is analogous to the proof of the upper and lower solutions ordering from(Nefedov, 1995).

\section{Conclusion}

In the paper we had examined a simple but well analyzed example. It shows that the asymptotical method of differential inequalities can be used not only to prove the existence and stability of solutions to boundary value problems with internal transition layers, but also to establish non-local domains of attractions of stable stationary solutions. This information can be quite useful for numerical researches especially for multidimensional problems for which the current study must be extended in the future. 


\section{References}

Inhomogeneous burgers equation with modular nonlinearity: Excitation and evolution of high-intensity waves. (2017). Doklady Mathematics, 95(3), 291-294.

On front motion in a burgers-type equation with quadratic and modular nonlinearity and nonlinear amplification. (2018). Doklady Mathematics, 97(1), 99-103.

Two approaches to describing the turbulent exchange within the atmospheric surface layer. (2017). Mathematical Models and Computer Simulations, 9(6), 697-707.

The use of contrast structures theory for the mathematical modelling of the wind field in spatially heterogeneous vegetation cover. (2017). Lecture Notes in Computer Science, 10187, 464-473.

A numerical method for a nonlinear singularly perturbed interior layer problem using an approximate layer location. (2015). Computational and Applied Mathematics, 290(15), 500-515.

Stabilised approximation of interior-layer solutions of a singularly perturbed semilinear reaction-diffusion problem. (2011). Numerische Mathematik, 119(2), 787-810.

Numerical method for a nonlinear singularly perturbed interior layer problem. (2011). Lectures Notes in Computational Science and Engeneering, 81, 187-195.

Solving of the coefficient inverse problems for a nonlinear singularly perturbed reaction-diffusion-advection equation with the final time data. (2018). Communications in Nonlinear Science and Numerical Simulation, $54,233-247$.

Asymptotic analysis of solving an inverse boundary value problem for a nonlinear singularly perturbed timeperiodic reaction-diffusion-advection equation. (2019). Journal of Inverse and Ill-Posed Problems., 27(5), $745-758$.

Solving of the coefficient inverse problem for a nonlinear singularly perturbed two-dimensional reactiondiffusion equation with the location of moving front data. (2019). Computers and Mathematics with Applications, 77(5), 1245-1254.

Asymptotics of the front motion in the reaction-diffusion-advection problem. (2014). Computational Mathematics and Mathematical Physics, 54(10), 1536-1549.

Step-like contrasting structures for a singularly perturbed quasilinear second-order differential equation. (1995). Comput. Math. Math. Phys., 35(4), 411-419.

Invariant criteria for existence of solutions to second-order quasilinear elliptic equations. (1978). Comm. Pure Appl. Math., 31(5), 619-645.

Monotone method for diffusion equations with nonlinear diffusion coefficients. (1998). Nonlinear Analysis, $34,113-142$.

The method of differential inequalities for some classes of nonlinear singularly perturbed problems with internal layers. (1995). Differential Equations, 31(7), 1077-1085.

Singularly perturbed problems with boundary and internal layers. (2010). Proc. Steklov Inst. Math., 268(1), $258-273$.

Existence and asymptotic stability of periodic solutions with an interior layer of reaction-advection-diffusion equations. (2013). J. Math. Anal. Appl., 409, 90-103.

The Boundary Function Method for Singular Perturbation Problems. (1995). SIAM.

Nonlinear Parabolic and Elliptic Equations. (1992). Plenum Press. 